\title{
Projectile dependence of single-excitation cross sections at intermediate velocities
}

\author{
A. Macías, F. Martín, A. Riera, and J. L. Sanz \\ Departamento de Química, C-9, Universidad Autónoma de Madrid, 28049-Madrid, Spain
}

(Received 16 October 1995)

\begin{abstract}
We study $1 s \rightarrow 2 s(2 p)$ excitation of hydrogen as a function of the nuclear charge of the projectile, $Z_{P}$, for a fixed impact velocity, $v=1.3$ a.u., for which electron excitation, capture, and ionization are competitive processes. For this purpose, we have performed large ab initio close-coupling calculations using a molecular basis in the framework of the impact-parameter method. Excitation probabilities do not saturate when $Z_{P}$ increases indefinitely, in agreement with recent work of Janev and with theoretical evidence on the behavior of transition probabilities at high impact energies.
\end{abstract}

PACS number(s): 34.50.Fa

Charge dependence of single excitation cross sections in ion-atom collisions, at a fixed impact velocity, is a controversial subject that has received a renewed attention in the last few years [1]. Many experiments have studied projectile excitation at intermediate energy using neutral targets [2-5]. The measured Lyman $\mathrm{x}$-ray cross sections, which are related to projectile excitation, are found to increase more slowly than predicted by the first Born approximation when the value of the target nuclear charge, $Z_{T}$, is larger than that of the projectile, $Z_{P}$. This slow increase of the cross sections has been referred to in the literature as saturation. However, from the measurement of Lyman x-ray cross sections it is difficult to analyze the excitation process itself, and to conclude whether excitation cross sections do exhibit saturation or not. As shown by Lüdde et al. [6], with a many-electron approach using the formalism of inclusive probabilities. Lyman x-ray emission cross sections do not only correspond to single excitation but to an ensemble of (many-electron) processes leading to $\mathrm{x}$-ray emission in the same frequency region of the spectrum. Moreover, in the range of intermediate impact velocities, target electrons have been found to play a crucial role in the production of radiative singly excited configurations. Although theoretical methods [2-4,7] based on the one-electron model yield results in good agreement with experiment, it has been found in [6] that the one-electron model breaks down for $Z_{T} \geqslant Z_{P}$.

In a different type of experiment involving high-energy collisions of bare nuclei with $\mathrm{H}$ and $\mathrm{He}$ targets [8,9], analysis of the excitation process in terms of the Janev-Presnyakov scaling rule [10] indicates that excitation cross sections do not saturate as a function of $q=Z_{P} / Z_{T}$. Recently, and using an analytical fit to measured cross sections for $q>1$ in terms of scaled energies, Janev [11] has predicted that $1 s \rightarrow 2 p$ excitation cross sections present a maximum at

$$
q^{\max }=2.8125 E(\mathrm{keV} / \mathrm{amu}) /\left(150 \omega_{2 p}\right)
$$

where $\omega_{2 p}$ is the transition energy and $E$ is the collision energy. This maximum corresponds to larger values of $q$ than those considered in $[2-4,8,9]$.

\footnotetext{
*Also at Instituto de Estructura de la Materia, Consejo Superior de Investigaciones, Científicas, Serrano 119, 28006 Madrid, Spain.
}

In the same line, Martín and Salin [12] have calculated excitation probabilities in collisions between high-energy bare ions and He using a one-center expansion of the electronic wave function. They found (see also [13]) that for a given impact parameter, the excitation probability passes through a maximum. We have integrated these probabilities for $v=2$ a.u. and the resulting cross sections present a maximum for $q=2$, in agreement with the value $\left(q^{\max }=2.3\right)$ obtained from (1).

The present work attempts to clarify the behavior of excitation cross sections by studying collisions of bare ions with hydrogen targets, thus eliminating many electron processes. We have chosen a nuclear velocity $v=1.3$ a.u. and $0.11<q<3$, so that values of $q>q^{\max }$ of (1) are considered. At this velocity and for these systems capture and ionization compete with excitation and must be taken into account in the theory. For H-like target ions of charge $Z_{T} \neq 1$, the corresponding cross sections (with $Z_{P}=q Z_{T}$ and for a velocity $\left.v=1.3 \times Z_{T}\right)$ are obtained by dividing our data by $Z_{T}^{2}$. The $q$ range analyzed in this work corresponds to the one recently used in [5] to study $\mathrm{Ar}^{16+}$ excitation with various neutral targets.

We solved the time-dependent Schrödinger equation in the impact-parameter approximation, for the nonrelativistic one-electron-diatomic molecule (OEDM) Hamiltonian.

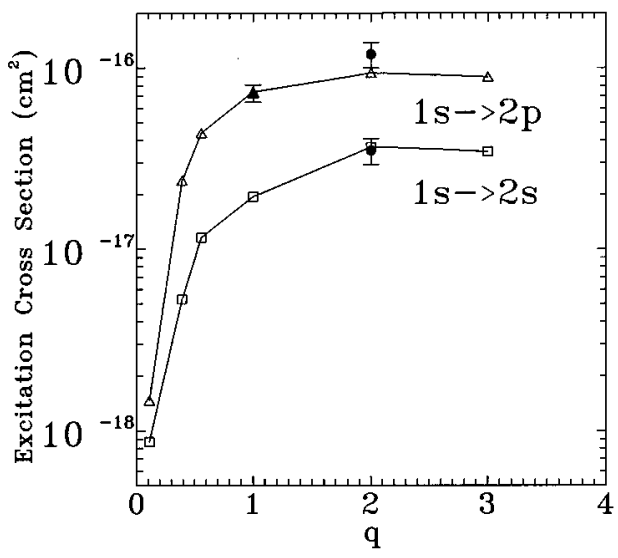

FIG. $1.2 s$ and $2 p$ excitation cross sections vs $q$. Empty squares and triangles: present results. Full circles: experimental values of Hughes et al. [20]. Full triangle: experimental value of Schartner et al. [19]. 
TABLE I. Basis sets and excitation cross sections. $q=Z_{P} / Z_{T} ; N_{t}$, number of OEDM orbitals included in the calculation associated to excitation channels; $N_{p}$, the same for charge-exchange channels; $n_{t}$, highest atomic principal quantum number for the excitation channels; $n_{p}$, highest atomic principal quantum number for charge-exchange channels, $\sigma_{2 s}\left(\sigma_{2 p}\right): 1 s-2 s(2 p)$ excitation cross sections in $\mathrm{cm}^{2}$. [-x] means $10^{[-x]}$.

\begin{tabular}{cccccc}
\hline \hline$q$ & $N_{t} / N_{p}$ & $n_{t}$ & $n_{p}$ & $\sigma_{2 s}$ & $\sigma_{2 p}$ \\
\hline 0.11 & $35 / 1$ & 5 & 1 & $8.69[-19]$ & $1.47[-16]$ \\
0.39 & $35 / 10$ & 5 & 3 & $5.30[-18]$ & $2.39[-17]$ \\
0.56 & $35 / 20$ & 5 & 4 & $1.16[-17]$ & $4.40[-17]$ \\
1 & $35 / 35$ & 5 & 5 & $1.99[-17]$ & $7.40[-17]$ \\
2 & $20 / 84$ & 4 & 7 & $3.76[-17]$ & $9.54[-17]$ \\
3 & $10 / 165$ & 3 & 9 & $3.45[-17]$ & $8.96[-17]$ \\
\hline \hline
\end{tabular}
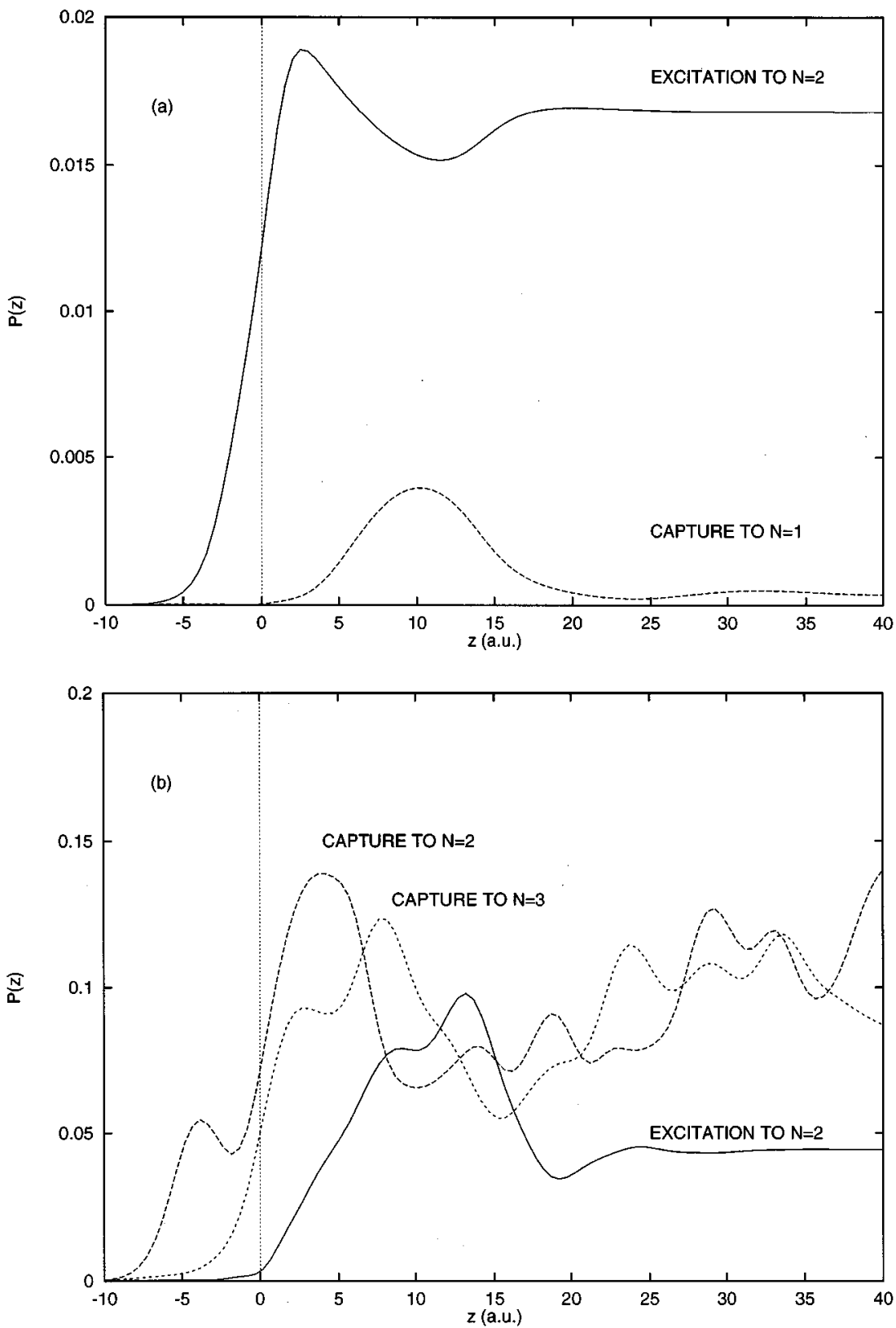

FIG. 2. Transition probabilities vs $z=v t$ for $b=2.5$ a.u. (a) $q=0.39$, (b) $q=2$. 

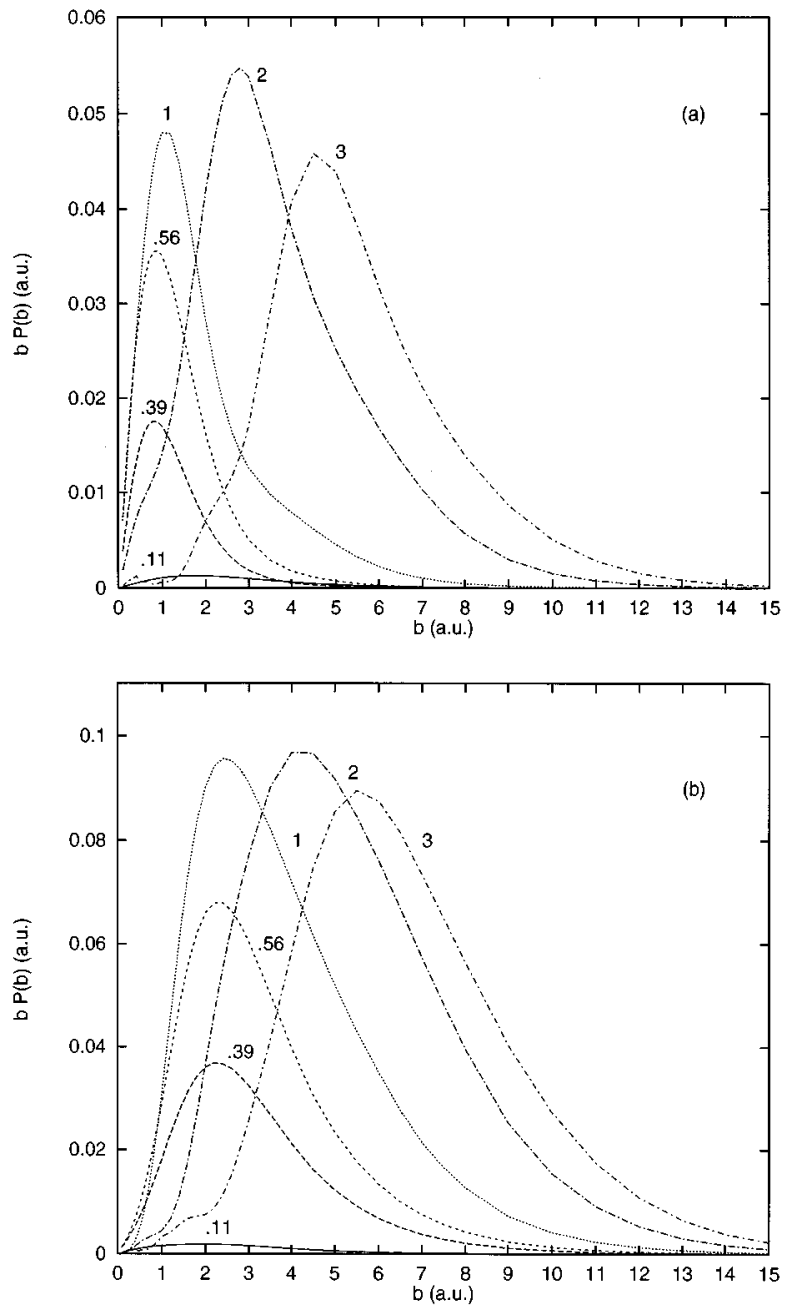

FIG. 3. Excitation probabilities (a) $b P_{2 s}$ and (b) $b P_{2 p}$ vs $b$. Numbers indicate values of $q$.

The scattering wave function was expanded in terms of the exact adiabatic eigenstates of the one-electron Hamiltonian (OEDM orbitals). Energies of and dynamical couplings between OEDM states were evaluated with the programs GRAVE and MEDOC [14]. The set of coupled equations for the expansion coefficients was solved with the program SUPERPAMPA [15]. As we are interested in target excitation cross sections, the origin of electronic coordinates was placed on the target nucleus [16]; then excitation amplitudes are given by the squares of the expansion coefficients associated to excitation channels. Ionization is taken into account in this treatment by including highly excited OEDM orbitals in the dynamical basis [17] which absorbs most of the ionization flux. The OEDM basis was increased until the $1 s \rightarrow 2 s, 2 p$ excitation cross sections converged; this procedure is justified because we are dealing with inner-shell $1 s \rightarrow 2 s(2 p)$ excitations, and cross sections may be expected to approach the exact results in a similar way to [17].

The basis sets used for each value of $Z_{P}$ is given in Table I. A large number of OEDM orbitals is needed to achieve convergence when $q \geqslant 1$ (up to 175 wave functions for $q=3$ ) for two reasons: first, the importance of ionization increases with $Z_{P}$, so that more OEDM orbitals are needed to describe the ionization flux; second, the number of capture channels

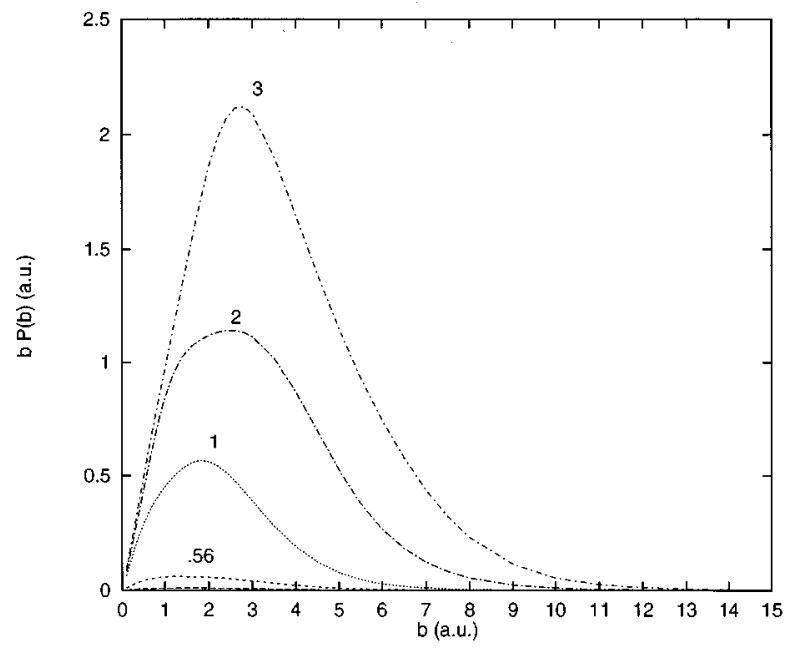

FIG. 4. Total electron-capture probabilities $b P_{\text {tec }}$ vs $b$. Numbers indicate values of $q$.

lying asymptotically between the entrance and the exit channels varies from zero for $q=0.11$, to 25 for $q=3$. Table I also includes the calculated $2 s$ and $2 p$ excitation cross sections for $v=1.3$ a.u., which have been plotted vs $q$ in Fig. 1. For $q<1$, excitation proceeds mainly through a rotational coupling between the entrance and the exit channels, and capture has a minor relevance [see Fig. 2(a)]; in contrast, for $q \geqslant 1$, the direct process via rotational coupling is less efficient because capture states are dominantly populated in the way-in of the collision and feed excitation channels in the way-out [see Fig. 2(b)].

Figure 1 and Table I show that there is a maximum in both the $2 s$ and $2 p$ excitation cross sections around $q=2$ and that they decrease slowly for larger values of $q$. For $v=1.3$ a.u. the agreement of our results with the cross sections predicted by Janev [11] (to an overall factor of 1.7), and with the value $q^{\max } \simeq 2$, obtained from (1) is excellent. Notice that from this equation the maximum for high collision energies obtains for much larger values of $q$, that is, for very asymmetrical systems.

Although there are no experimental results for the whole series of $q$ values considered in this work, Schartner et al. [18] have recently reported $\sigma_{2 p}$ for $\mathrm{H}^{+}+\mathrm{H}(1 s)$ and Hughes et al. [19], $\sigma_{2 s}$ and $\sigma_{2 p}$ for $\mathrm{He}^{2+}+\mathrm{H}(1 s)$ at $E=40 \mathrm{keV} / \mathrm{amu}$, which is very close to the impact energy considered here. These experimental cross sections, included in Fig. 1, are in excellent agreement with our calculated values. Moreover, the agreement between the present $\sigma_{2 p}$ cross section (scaled in the way mentioned above) with the Lyman x-ray cross sections reported in [5] for $q<1$ (where a meaningful comparison with a one-electron model is allowed, see Ref. [6]) is also very good.

In Fig. 3 we have plotted the transition probabilities multiplied by the impact parameter $b P_{2 s}$ and $b P_{2 p}$ as functions of $b$. For $q \leqslant 1$ all these probabilities exhibit a maximum around $b=1$ a.u. (for $2 s$ excitation) and $b=2.5$ a.u. (for $2 p$ excitation) and the magnitude of this maximum increases with $q$. For $q>1$ the mechanism changes: the probability decreases at small impact parameters, the position of the maximum shifts to larger impact parameters, and its magni- 
tude decreases. This behavior is identical to that observed in [12] at higher energies, except that now the transition from one mechanism to the other takes place at smaller values of $q$. For the collision velocities investigated in [12], charge exchange is small or negligible and the decrease of excitation probabilities at small impact parameters is exclusively due to an enhancement of ionization for large $q$. Although the decrease of the excitation probability in the present case is due to both electron capture and ionization, it was shown [17] that in the molecular picture ionization proceeds via charge exchange channels, so that the general behavior of the excitation probabilities is similar to that found at higher energies. Since the number of states which are significantly populated at small impact parameters increases with $q$, the probability describing excitation to a given state decreases. This explanation is supported by the fact that "total electron-capture (tec)" probabilities, $b P_{\text {tec }}(b)$, increase with $q$ in the whole range of $q$ values investigated here and exhibit a maximum at lower impact parameters than single excitation probabilities (see Fig. 4).
In summary, we have performed large $a b$ initio closecoupling calculations of excitation cross sections at intermediate velocities using a molecular expansion of the electronic wave function in the impact-parameter approximation. Our results show that target excitation cross sections increase with projectile charge up to $q \simeq 1$, where they present a maximum, and then slowly decrease for larger values of this ratio. For small $q$ values excitation is governed by a direct transition between the (rotationally coupled) entrance and exit channels, whereas for larger $q$ capture states populated in the way in of the collision share part of their population with excitation channels in the way out. Consequently, excitation cross sections do not "saturate," in the sense that they do not become strictly constant, in agreement with [11]; we also confirm the behavior of excitation probabilities recently found in [12] at higher collision velocities.

The authors acknowledge useful discussions with R. Gayet, H. J. Lüdde, and A. Salin. This work was partially supported by the DGICYT Project Nos. PB93-0288-C02-01 and 02.
[1] K. Reyman, K. H. Schartner, and B. Sommer, Phys. Rev. A 38, 2290 (1988); V. D. Rodríguez, J. Phys. B 24, L205 (1991); V. D. Rodríguez and J. E. Miraglia, ibid. 23, 3629 (1990); 25, 2037 (1992); C. O. Reinhold, R. E. Olson, and W. Fritsch, Phys. Rev. A 41, 4837 (1990).

[2] B. Brendlé, R. Gayet, J. P. Rozet, and K. Wohrer, Phys. Rev. Lett. 54, 2007 (1985).

[3] K. Wohrer, A. Chetioui, J. P. Rozet, A. Jolly, F. Fernandez, C. Stephan, B. Brendlé, and R. Gayet, J. Phys. B 19, 1997 (1986).

[4] M. Chabot, K. Wohrer, A. Chetioui, J. P. Rozet, A. Touati, D. Vern, M. F. Politis, C. Stephan, J. P. Grandin, A. Macías, F. Martín, A. Riera, J. L. Sanz, and R. Gayet, J. Phys. B 27, 111 (1994).

[5] L. Adoui, D. Vernhet, K. Wohrer, J. Plante, A. Chetioui, J. P. Rozet, I. Despiney, C. Stephan, A. Touati, J. M. Ramillon, A. Cassimi, J. P. Grandin, and M. Cornille, Nucl. Instrum. Methods Phys. Res. B 98, 312 (1995).

[6] H. J. Ludde, A. Macías, F. Martín, A. Riera, and J. L. Sanz, J. Phys. B 28, 4101 (1995).
[7] G. H. Olivera, V. D. Ramírez, and R. D. Rivarola, Phys. Rev. A 47, 1000 (1993).

[8] M. Anton, D. Detleffsen, K. -H. Schartner, and A. Werner, J. Phys. B 26, 2005 (1993).

[9] D. Detleffsen, M. Anton, A. Werner, and K.-H. Schartner, J. Phys. B 27, 4195 (1994).

[10] R. K. Janev and L. P. Presnyakov, J. Phys. B 13, 4233 (1980).

[11] R. K. Janev (unpublished); Phys. Rev. A 53, 219 (1996).

[12] F. Martín and A. Salin, J. Phys. B 28, 671 (1995).

[13] V. D. Rodríguez and A. Salin, J. Phys. B 25, L467 (1992).

[14] A. Salin, Comput. Phys. Commun. 14, 121 (1978).

[15] A. Salin, Comput. Phys. Commun. 62, 58 (1991).

[16] R. D. Piacentini and A. Salin, J. Phys. B 7, 1666 (1974).

[17] L. F. Errea, C. Harel, H. Jouin, L. Méndez, B. Pons, and A. Riera, J. Phys. B 27, 3603 (1994).

[18] H. A. Slim and A. M. Ermolaev, J. Phys. B 27, L203 (1994). See K.-H. Schartner et al. (private communication).

[19] M. P. Hughes, J. Geddes, and H. B. Gilbody, J. Phys. B 27, 1143 (1994). 